\title{
Correction to: Failure Analysis of a GTD-111 Turbine Blade Using Metallurgical Analysis and Fractography
}

\author{
Esmaeil Poursaeidi - Morteza Aieneravaie - Reza Bannazadeh • \\ Kaveh Torkashvand
}

Published online: 18 October 2019

(c) ASM International 2019

Correction to: J Fail. Anal. and Preven. https://doi.org/10.1007/s11668-019-00732-9

In the title for Table 5, "region A in Fig. 6b" should read "region A in Fig. 7b."

In the title for Table 6, "region B in Fig. 6b" should read "region B in Fig. 7b."

In the title for Table 7, "region $\mathrm{C}$ in Fig. 6b" should read "region C in Fig. 7b."

In the title for Table 8, "region E in Fig. 8" should read "region E in Fig. 9."

In the title for Table 9, "region $\mathrm{F}$ in Fig. 8" should read "region F in Fig. 9."

In the title for Table 10, "region G in Fig. 8" should read "region G in Fig. 9."

In the title for Table 11, "region $\mathrm{H}$ in Fig. 8" should read "region $\mathrm{H}$ in Fig. 9."

In the title for Table 12, "region E in Fig. 10" should read "region E in Fig. 11."
In the title for Table 13, "region A in Fig. 14" should read "region A in Fig. 15."

In the title for Table 14, "region B in Fig. 15" should read "region B in Fig. 16."

In the title for Table 15, "region $\mathrm{C}$ in Fig. 15" should read "region C in Fig. 16."

In the title for Table 16, "region D in Fig. 16" should read "region D in Fig. 17."

In the section "Metallographic Examination and Microprobe Analysis," the sentence "Also, the chemical composition of region $\mathrm{H}$ shows that this region and region $\mathrm{G}$ are similar and are formed during the cooling process" should read "Also, the chemical composition of region $\mathrm{H}$ shows that this region and region $\mathrm{G}$ are similar and are formed during the solidification process [29]."

Publisher's Note Springer Nature remains neutral with regard to jurisdictional claims in published maps and institutional affiliations.

The original article can be found online at https://doi.org/10.1007/ s11668-019-00732-9.

E. Poursaeidi $(\bowtie) \cdot K$. Torkashvand

Department of Mechanical Engineering, University of Zanjan,

Zanjan, Iran

e-mail: epsaeidi@gmail.com

M. Aieneravaie $\cdot$ R. Bannazadeh

IPPR Research Lab, Iran Power Plant Repairs Co., Karaj, Iran 